R. F. de Oliveira

J. A. F. Santiago and F. Cezario COPPE/UFRJ

Programa de Engenharia Civil

Cx. Postal 68519

21945-970 Rio de Janeiro, RJ. Brazil

roberto@coc.ufrj.br

santiago@coc.ufrj.br

and cezario@coc.ufrj.br

\section{Novozhilov's Mean Rotation Measures Invariance}

In this article the invariance of the Novozhilov's mean rotation measures will be emphasize by the invariance of the determinant of the gradient of deformation tensor of continuum mechanics, or the invariance of the second principal invariant of the tensor $(\mathbf{I}+\mathbf{E})^{-1} \mathbf{W}$. Keywords: mean rotation measure, finite rotation, finite deformation, Novozhilov's mean rotation, linear algebra

\section{Introduction}

In the fourth decade of the 20th century Novozhilov obtained a measure of the mean rotation (Novozhilov, 1971) by modifying a previous definition produced by Cauchy (Cauchy, 1841). In the literature, this measure has been named Novozhilov's mean rotation measure ever since.

The original Novozhilov's expression was acquired in terms of Cartesian coordinates, therefore hiding its invariant character.

Later on Truesdell and Toupin (Truesdell and Toupin, 1960) reevaluated Novozhilov's measure and found there was a hidden invariance in it.

The present article enhances the invariance of Novozhilov's mean rotation measure. This invariance is derived through the invariance of the determinant of the gradient of the deformation tensor $\mathbf{F}$ (Continuum Mechanics), or even the invariance of the second invariant of the tensor $(\mathbf{I}+\mathbf{E})^{-1} \mathbf{W}$, when the Cartesian decomposition $\mathbf{F}=\mathbf{I}+\mathbf{E}+\mathbf{W}$ is adopted for the tensor $\mathbf{F}$.

An arbitrary tensor $\mathbf{S}$ can be decomposed, among other ways, in the following: (i) polar decomposition $\mathbf{S}=\mathbf{R} \mathbf{U}, \mathbf{R} \in$ Orth, $\mathbf{U} \in \mathrm{Sym}$; (ii) Cartesian decomposition, $\mathbf{S}=\operatorname{sym} \mathbf{S}+\operatorname{skw} \mathbf{S}$ and (iii) additive decomposition, $\mathbf{S}=\mathbf{E}_{\mathbf{s}}+\mathbf{D}_{\mathbf{s}}$, being $\mathbf{E}_{\mathbf{s}}$ the spherical tensor of $\mathbf{S}$ and $\mathbf{D}_{\mathbf{s}}$ the deviator tensor of $\mathbf{S}$ (according to Coimbra, 1981, for example), which is a null trace tensor.

The distributivity of the determinant of $\mathbf{S}$, $\operatorname{det} \mathbf{S}$, in the polar decomposition is valid, while in the additive and Cartesian decompositions it is not valid in general (particularly when the domain of $\mathbf{S}$ has dimension two, the distributivity of $\operatorname{det} \mathbf{S}$ is valid). When $\operatorname{dim}($ domain $\mathbf{S})=3$ and $\mathbf{S}$ is the gradient of the deformation tensor $\mathbf{F}$, from the Continuum Mechanics, the $\operatorname{det} \mathbf{F}$ involves the measures of Novozhilov's mean rotation, whose invariance is due to the invariance of detF.

\section{Determinant of the Gradient of the Deformation}

Let the gradient of deformation tensor be denoted by $\mathbf{F}: \mathbf{V} \rightarrow \mathbf{V}$, $\operatorname{dim} \mathbf{V}=3$, and consider its Cartesian decomposition $\mathbf{F}=\mathbf{I}+\mathbf{E}+\mathbf{W}$, with $(\mathbf{I}+\mathbf{E}) \in$ Sym and $\mathbf{W} \in \mathrm{Skw}^{1}$. Hence the determinant of $\mathbf{F}$ is calculated as:

$$
\operatorname{det} \mathbf{F}=\operatorname{det}(\mathbf{I}+\mathbf{E}+\mathbf{W})
$$

\footnotetext{
Paper accepted June, 2005. Technical Editor: Atila P. Silva Freire.
}

${ }^{1}$ The notations used in this article are the continuum mechanics usual ones, found for example in (Gurtin, 1981).
Supposing that $\operatorname{det}(\mathbf{I}+\mathbf{E}) \neq 0$, the previous expression can be rewritten as:

$$
\begin{aligned}
\operatorname{det} \mathbf{F} & =\operatorname{det}\left[(\mathbf{I}+\mathbf{E})+(\mathbf{I}+\mathbf{E})(\mathbf{I}+\mathbf{E})^{-1} \mathbf{W}\right] \\
& =\operatorname{det}(\mathbf{I}+\mathbf{E}) \operatorname{det}\left[\mathbf{I}+(\mathbf{I}+\mathbf{E})^{-1} \mathbf{W}\right]
\end{aligned}
$$

It is known that the characteristic polynomial of any tensor $\mathbf{S} \in$ Lin is given by

$$
\operatorname{det}(\mathbf{S}-\lambda \mathbf{I})=-\lambda^{3}+(\operatorname{tr} \mathbf{S}) \lambda^{2}-\mathrm{II}_{\mathbf{S}} \lambda+\operatorname{det} \mathbf{S},
$$

where $\mathrm{II}_{\mathbf{S}}$ is the second principal invariant of $\mathbf{S}$. Substituting $\lambda=-1$ in the expression (2a), the following expression is obtained:

$$
\operatorname{det}(\mathbf{I}+\mathbf{S})=1+\operatorname{tr} \mathbf{S}+\mathrm{II}_{\mathbf{S}}+\operatorname{det} \mathbf{S} .
$$

Introducing the expression (2b) in the equation (1), and considering $\mathbf{S}=(\mathbf{I}+\mathbf{E})^{-1} \mathbf{W}$ gives:

$$
\operatorname{det} \mathbf{F}=\operatorname{det}(\mathbf{I}+\mathbf{E})\left\{1+\operatorname{tr}\left[(\mathbf{I}+\mathbf{E})^{-1} \mathbf{W}\right]+\mathbf{I I}_{(\mathbf{I}+\mathbf{E})^{-1} \mathbf{W}}\right\},
$$

taking into consideration that $\operatorname{det}\left[(\mathbf{I}+\mathbf{E})^{-1} \mathbf{W}\right]=\operatorname{det}(\mathbf{I}+\mathbf{E})^{-1} \operatorname{det} \mathbf{W}=$ 0 because $\mathbf{W} \in$ Skw. Keeping in mind that $\operatorname{tr}\left[(\mathbf{I}+\mathbf{E})^{-1} \mathbf{W}\right]=(\mathbf{I}+$ $\mathbf{E})^{-1} \bullet \mathbf{W}=0$ (result of the inner product of a symmetric tensor by an skew symmetric one), the expression of $\operatorname{det} \mathbf{F}$ can still be written as:

$$
\operatorname{det} \mathbf{F}=\operatorname{det}(\mathbf{I}+\mathbf{E})\left[1+\mathrm{II}_{(\mathbf{I}+\mathbf{E})}{ }^{-1} \mathbf{w}\right] .
$$

Taking account of that $\mathrm{II}_{\mathbf{S}}=\frac{1}{2}\left[(\operatorname{tr} \mathbf{S})^{2}-\operatorname{tr}\left(\mathbf{S}^{2}\right)\right]$, the invariant $\mathrm{II}_{(\mathbf{I}+}$ E) ${ }^{-1} \mathbf{w}$ can be written as:

$$
\begin{aligned}
\operatorname{II}_{(\mathbf{I}+\mathbf{E})^{-1} \mathbf{W}=\frac{1}{2}\{}\left\{\left(\operatorname{tr}\left[(\mathbf{I}+\mathbf{E})^{-1} \mathbf{W}\right]\right)^{2}-\operatorname{tr}\left[(\mathbf{I}+\mathbf{E})^{-1} \mathbf{W}(\mathbf{I}+\mathbf{E})^{-1} \mathbf{W}\right]\right\} \\
=\frac{1}{2} \operatorname{tr}\left[\mathbf{W}^{\mathrm{T}}(\mathbf{I}+\mathbf{E})^{-1} \mathbf{W}(\mathbf{I}+\mathbf{E})^{-1}\right] \\
=\frac{1}{2} \mathbf{W} \cdot\left[(\mathbf{I}+\mathbf{E})^{-1} \mathbf{W}(\mathbf{I}+\mathbf{E})^{-1}\right]
\end{aligned}
$$

Since $(\mathbf{I}+\mathbf{E})^{-1} \in$ Sym, its spectral decomposition is considered, according to (Gurtin, 1981), as: 
$(\mathbf{I}+\mathbf{E})^{-1}=\frac{1}{1+\varepsilon_{1}} \mathbf{e}_{1} \otimes \mathbf{e}_{1}+\frac{1}{1+\varepsilon_{2}} \mathbf{e}_{2} \otimes \mathbf{e}_{2}+\frac{1}{1+\varepsilon_{3}} \mathbf{e}_{3} \otimes \mathbf{e}_{3}$, being

$\varepsilon_{1}, \varepsilon_{2}, \varepsilon_{3}$ - principal elongations of $\mathbf{E}$,

$\mathbf{e}_{1}, \mathbf{e}_{2}, \mathbf{e}_{3}$ - principal directions of elongations of $\mathbf{E}$.

Taking this expression in equation (4) and after some algebraic operations gives

$$
\frac{1}{2} \mathbf{W} \bullet\left[\begin{array}{c}
\mathrm{II}_{(\mathbf{I}+\mathbf{E})}{ }^{-1} \mathbf{W}= \\
-\frac{\mathbf{e}_{1} \bullet \mathbf{W} \mathbf{e}_{2}}{\left(1+\varepsilon_{1}\right)\left(1+\varepsilon_{2}\right)}\left(\mathbf{e}_{2} \otimes \mathbf{e}_{1}-\mathbf{e}_{1} \otimes \mathbf{e}_{2}\right)+ \\
\frac{\mathbf{e}_{1} \bullet \mathbf{W} \mathbf{e}_{3}}{\left(1+\varepsilon_{1}\right)\left(1+\varepsilon_{3}\right)}\left(\mathbf{e}_{1} \otimes \mathbf{e}_{3}-\mathbf{e}_{3} \otimes \mathbf{e}_{1}\right) \\
-\frac{\mathbf{e}_{2} \bullet \mathbf{W} \mathbf{e}_{3}}{\left(1+\varepsilon_{2}\right)\left(1+\varepsilon_{3}\right)}\left(\mathbf{e}_{3} \otimes \mathbf{e}_{2}-\mathbf{e}_{2} \otimes \mathbf{e}_{3}\right)
\end{array}\right] .
$$

\section{Novozhilov's Mean Rotation Measures}

Denoting by

$\mathbf{Z}_{3}:=\mathbf{e}_{2} \otimes \mathbf{e}_{1}-\mathbf{e}_{1} \otimes \mathbf{e}_{2}-$ skew symmetric tensor of axial vector $\mathbf{e}_{3}$, $\mathbf{Z}_{2}:=\mathbf{e}_{1} \otimes \mathbf{e}_{3}-\mathbf{e}_{3} \otimes \mathbf{e}_{1}-$ skew symmetric tensor of axial vector $\mathbf{e}_{2}$, $\mathbf{Z}_{1}:=\mathbf{e}_{3} \otimes \mathbf{e}_{2}-\mathbf{e}_{2} \otimes \mathbf{e}_{3}-$ skew symmetric tensor of axial vector $\mathbf{e}_{1}$,

equation (5) can be written as:

$$
\mathrm{II}_{(\mathbf{I}+\mathbf{E})}{ }^{-1} \mathbf{w}=\frac{1}{2} \mathbf{W} \bullet\left[\begin{array}{c}
-\frac{\mathbf{e}_{1} \bullet \mathbf{W} \mathbf{e}_{2}}{\left(1+\varepsilon_{1}\right)\left(1+\varepsilon_{2}\right)} \mathbf{Z}_{3}+ \\
\frac{\mathbf{e}_{1} \bullet \mathbf{W} \mathbf{e}_{3}}{\left(1+\varepsilon_{1}\right)\left(1+\varepsilon_{3}\right)} \mathbf{Z}_{2}- \\
\frac{\mathbf{e}_{2} \bullet \mathbf{W e} \mathbf{e}_{3}}{\left(1+\varepsilon_{2}\right)\left(1+\varepsilon_{3}\right)} \mathbf{Z}_{1}
\end{array}\right] .
$$

Denoting by $\omega$ the axial vector of $\mathbf{W}$, the following expressions may be obtained:

$$
\begin{gathered}
\mathbf{e}_{2} \bullet \mathbf{W} \mathbf{e}_{3}=\mathbf{e}_{2} \bullet\left(\omega \times \mathbf{e}_{3}\right)=-\omega \bullet\left(\mathbf{e}_{2} \times \mathbf{e}_{3}\right)=-\omega \bullet \mathbf{e}_{1}, \\
\mathbf{e}_{1} \bullet \mathbf{W} \mathbf{e}_{3}=\mathbf{e}_{1} \bullet\left(\omega \times \mathbf{e}_{3}\right)=\omega \bullet\left(\mathbf{e}_{3} \times \mathbf{e}_{1}\right)=\omega \bullet \mathbf{e}_{2}, \\
\mathbf{e}_{1} \bullet \mathbf{W} \mathbf{e}_{2}=\mathbf{e}_{1} \bullet\left(\omega \times \mathbf{e}_{2}\right)=-\omega \bullet\left(\mathbf{e}_{1} \times \mathbf{e}_{2}\right)=-\omega \bullet \mathbf{e}_{3} .
\end{gathered}
$$

Introducing these three expressions in equation (6) one obtains

$$
\begin{gathered}
\mathrm{II}_{(\mathbf{I}+\mathbf{E})^{-1} \mathbf{W}}=\frac{\omega \bullet \mathbf{e}_{1}}{2\left(1+\varepsilon_{2}\right)\left(1+\varepsilon_{3}\right)} \mathbf{W} \bullet \mathbf{Z}_{1}+\frac{\omega \bullet \mathbf{e}_{2}}{2\left(1+\varepsilon_{1}\right)\left(1+\varepsilon_{3}\right)} \mathbf{W} \cdot \mathbf{Z}_{2}+ \\
\frac{\omega \bullet \mathbf{e}_{3}}{2\left(1+\varepsilon_{1}\right)\left(1+\varepsilon_{2}\right)} \mathbf{W} \cdot \mathbf{Z}_{3} .
\end{gathered}
$$

Considering that $\mathbf{W} \bullet \mathbf{Z}_{\mathrm{i}}=2 \omega \bullet \mathbf{e}_{\mathrm{i}}, \mathrm{i}=1,2,3$, according to (Chadwick, 1999) comes:

$$
\mathrm{II}_{(\mathbf{I}+\mathbf{E})}{ }^{-1} \mathbf{w}=\frac{\left(\omega \bullet \mathbf{e}_{1}\right)^{2}}{\left(1+\varepsilon_{2}\right)\left(1+\varepsilon_{3}\right)}+\frac{\left(\omega \bullet \mathbf{e}_{2}\right)^{2}}{\left(1+\varepsilon_{1}\right)\left(1+\varepsilon_{3}\right)}+\frac{\left(\omega \bullet \mathbf{e}_{3}\right)^{2}}{\left(1+\varepsilon_{1}\right)\left(1+\varepsilon_{2}\right)} .
$$

Using the notation of (Oliveira, 1987) the terms of the expression of $\mathrm{II}_{(\mathbf{I}+\mathbf{E})}{ }^{-1} \mathbf{W}$ can be written as:

$$
\frac{\omega \bullet \mathbf{e}_{1}}{\sqrt{\left(1+\varepsilon_{2}\right)\left(1+\varepsilon_{3}\right)}}=\mathrm{C}\left(\mathbf{F} ; \mathbf{e}_{1}\right)-\text { Novozhilov's mean rotation }
$$

measure, around $\mathbf{e}_{1}$; $\frac{\omega \bullet \mathbf{e}_{2}}{\sqrt{\left(1+\varepsilon_{1}\right)\left(1+\varepsilon_{3}\right)}}=\mathrm{C}\left(\mathbf{F} ; \mathbf{e}_{2}\right)-$ Novozhilov's mean rotation measure, around $\mathbf{e}_{2}$;

$\frac{\omega \bullet \mathbf{e}_{3}}{\sqrt{\left(1+\varepsilon_{1}\right)\left(1+\varepsilon_{2}\right)}}=\mathrm{C}\left(\mathbf{F} ; \mathbf{e}_{3}\right)-$ Novozhilov's mean rotation measure, around $\mathbf{e}_{3}$,

which gives :

$$
\begin{gathered}
\left.\mathrm{II}_{(\mathbf{I}+\mathbf{E})}\right)^{-1} \mathbf{w}= \\
-\frac{1}{2} \operatorname{tr}\left[(\mathbf{I}+\mathbf{E})^{-2} \mathbf{W}^{2}\right]=\left[\mathrm{C}\left(\mathbf{F}, \mathbf{e}_{1}\right)\right]^{2}+\left[\mathrm{C}\left(\mathbf{F}, \mathbf{e}_{2}\right)\right]^{2}+\left[\mathrm{C}\left(\mathbf{F}, \mathbf{e}_{3}\right)\right]^{2}
\end{gathered}
$$

Substituting expression (7) in equation (3) it follows:

$\operatorname{det} \mathbf{F}=\operatorname{det}(\mathbf{I}+\mathbf{E})\left\{1+\left[\mathrm{C}\left(\mathbf{F}, \mathbf{e}_{1}\right)\right]^{2}+\left[\mathrm{C}\left(\mathbf{F}, \mathbf{e}_{2}\right)\right]^{2}+\left[\mathrm{C}\left(\mathbf{F}, \mathbf{e}_{3}\right)\right]^{2}\right\}$.

\section{Conclusions}

The following invariance of Novozhilov's mean rotation measures is inferred from expressions (7) and (8): the sum of the squares of Novozhilov's mean rotation measures, around three directions mutually orthogonal (specifically the second principal invariant of the tensor $\left.(\mathbf{I}+\mathbf{E})^{-1} \mathbf{W}\right)$.

At the proof of the results, the eigenvectors $\mathbf{e}_{1}, \mathbf{e}_{2}$ and $\mathbf{e}_{3}$ (the principal directions of elongation of the tensor $\mathbf{E}$ ) were used as base of $\mathbf{V}$, in order to make the expressions of the mean rotation $\mathrm{C}\left(\mathbf{F} ; \mathbf{e}_{\mathrm{i}}\right)$ simpler.

As $\mathrm{C}(\mathbf{F} ; \mathbf{n})$ is the average value of the tangents of the rotation angles of fibers of an elastic body around a given direction $\mathbf{n}$, the Novozhilov's mean rotation measure can fail in situations where the deformation of the body causes a $90^{\circ}$ or a $270^{\circ}$ rotation of a fiber around $\mathbf{n}$ (in this case $\mathrm{C}(\mathbf{F} ; \mathbf{n}) \rightarrow \infty$, as it occurs in Truesdell \& Toupin, 1960, page 293 , when $\mathrm{K}=2$ is used in the equation $(36.8)_{2}$ from page 276).

Another mean rotation measure, based on the mean angle of fiber rotations of a body around a direction $\mathbf{n}$, solves also the situation described above, being therefore valid for all possible deformations of the elastic body. It is called the Cauchy mean rotation measure and it has been developed in (Cauchy, 1841), (Oliveira, 1987), (Zheng \& Hwang, 1989 and 1992) and (Martins \& Podio-Guidugli, 1992).

\section{Acknowledgements}

The second and third authors would like to thank CNPq for providing financial support to this research.

\section{References}

Cauchy, A. L., 1841, "Mémoire sur les dilatations, les condensations et les rotations produits par un changement de forme dans un système de points matériels", Oeuvres (2)12, 343-377.

Chadwick, P., 1999, "Continuum Mechanics: concise theory and problems", Dover, Mineola, N. Y., U.S.A..

Coimbra, A. L., 1981, "New Lessons of Continuum Mechanics" (in Portuguese), Edgard Blücher Ltda., São Paulo, Brasil.

Gurtin, Morton E., 1981, "An Introduction to Continuum Mechanics", Academic Press, New York, U.S.A.

Martins, L. C. and Podio-Guidugli, P., 1992, "On the local measures of mean rotation in continuum mechanics", Journal of Elasticity, 27, 267-279.

Novozhilov, V. V., 1971, "Foundations of the Nonlinear Theory of Elasticity", fourth printing, Graylock, Rochester, N. Y., U.S.A..

Oliveira, R. F., 1987, "Local Analysis of Mean Rotation Measure in Continuum Mechanics" (in Portuguese), tese de D.Sc., COPPE/UFRJ, Rio de Janeiro, Brasil. 
Truesdell, C. A. and Toupin, R. A., 1960, "The Classical Field Theories", Handbuch der Physik, vol. III-1, Springer-Verlag, Berlin, Germany.
Zheng, Q.-S. and Hwang, K. C., 1989, "Cauchy's Mean Rotation", Chinese Science Bulletin, vol. 34, No. 11, 897-901.

Zheng, Q.-S. and Hwang, K. C., 1992, "On Cauchy's Mean Rotation", Journal of Applied Mechanics, Transactions of the ASME, vol. 59, 405-410. 